\title{
Factitious Pseudo-Membranous Conjunctivitis in an Adolescent Boy
}

\author{
Jelka G. Orsoni ${ }^{a}$ Pierangela Rubino ${ }^{a} \quad$ Isabella Pellistria \\ Chiara Menozzi $^{a} \quad$ Laura Zavota $^{b}$ Antonino Massaro ${ }^{c}$ \\ Paolo Mora ${ }^{a}$ \\ anstitute of Ophthalmology, University of Parma, and bepartment of Pediatrics, \\ Azienda Ospedaliero-Universitaria, Parma, and 'Local Ophthalmologist, \\ Agrigento, Italy
}

\section{Key Words}

Factitious pseudo-membranous conjunctivitis · Self-induced conjunctivitis - Anorexia . Cooperation between ophthalmologist and psychiatrist

\begin{abstract}
Background/Aims: Ocular factitious lesions involving the conjunctiva alone represent a challenging diagnosis for the ophthalmologist; corneal integrity, in fact, allows maintenance of good visual acuity and precludes the pain subsequent to trigeminal stimulation. Conjunctival biopsy is crucial to make a diagnosis and to focus on possible peculiarities in the patient's behavior. A psychiatrist has to confirm the diagnosis. In this case report, images of a bilateral pseudo-membranous conjunctivitis sparing the cornea in an anorexic adolescent boy are shown.
\end{abstract}

Methods: Photographically documented case report.

Results: A fourteen-year-old Italian boy was referred with a diagnosis of bilateral chronic conjunctivitis unresponsive to systemic and topical antibiotic and steroidal treatment. It had lasted for 4 months and was concomitant with an 8-kg weight loss. Conjunctival biopsy revealed cotton wool fragments. The patient admitted an unsafe behaviour lasting for months. A diagnosis of factitious conjunctivitis was made, and confirmed by a psychiatric assessment.

Conclusion: Factitious lesions of the eye involve not only anatomical structures situated on the visual axis causing a reduction of visual acuity, but may also involve the conjunctiva alone. A thorough clinical history should identify the source of the patient's anxiety. Moreover, close cooperation between ophthalmologists and a psychiatrist can further clarify the diagnosis. 


\section{Case Report}

A fourteen-year-old Italian boy was referred to our department with a diagnosis of bilateral conjunctivitis lasting 4 months, unresponsive to both systemic and topical antibiotic and steroid treatment. Recently, the boy had lost $8 \mathrm{~kg}$ without any apparent disease. Eye symptoms presented after a school trip to the woods, which prompted a search for caterpillar hairs in the anterior segment, not found on careful slit-lamp examination. During his first hospitalization, in another medical facility, a conjunctival biopsy was performed in both eyes. Histological examination revealed granulation tissue with eosinophilic and giant cells, reactive to foreign hairs. Conjunctival swabs for bacteria, Chlamydia, herpes and adenoviruses were negative. General clinical examinations, blood and chemical tests were normal. Infectious, autoimmune and neoplastic diseases of any kind that might explain the significant weight loss were excluded. Therefore, the clinician who first treated the patient suspected an inflammation due to contact with caterpillar hairs.

At the time of our visit, 4 months after symptoms onset, the boy weighed $38 \mathrm{~kg}$ and was $160 \mathrm{~cm}$ tall. His father was a baker and his mother a housewife. The grandparents own agricultural land and produce fruit and vegetables which they sell at the market. Over the previous few months, the boy's eating behavior had become particularly difficult, and he refused his father's and grandparents' products. According to his mother, the boy's weight loss was a consequence of his very low food intake. A diagnosis of bilateral pseudo-membranous conjunctivitis sparing the cornea was made. Furthermore, this explains the well-conserved visual acuity, 20/32 in right eye and 20/25 in left eye. Extremely intense conjunctival bulbar and conjunctival hyperemia and chemosis without any discharge were evident (fig. 1, fig. 2). Both corneas were fluorescein negative. Ocular motility and funduscopy were normal. We asked the initial treating clinic for the histological slides for a reexamination. Our own pathologist, unaware of the clinical history, formally excluded caterpillar hairs reporting the presence of birefrangent material typical of cotton wool. After carefully questioning the boy, he admitted having repeatedly rubbed both eyes with Kleenex over the last 4 months. Since all performed tests were negative, we suspected that the boy's weight loss was due to anorexia, which was confirmed by a psychiatrist. The family completely denied any kind of familial problem.

Systemic and topical anti-inflammatory treatments controlled the inflammation, but were ineffective on the membranes. Psychiatric treatment with the aim of resolving anorexia was suggested, but was only partially accepted by the parents. They eventually agreed to consult a psychiatrist far from the village where they live. Since the patient also lived at some distance from our hospital, we only managed to follow his clinical situation for a few months. The local ophthalmologist (A.M.) refers good visual acuity, the persistence of conjunctival membranes without inflammation, but persistent poor weight, borderline to anorexic.

\section{Comment}

Factitious disorders are conditions in which symptoms or physical findings are intentionally produced by the patient to feign illness [1]. Munchausen syndrome (MS) is probably the most popular amongst self-induced diseases already recognized in medical literature as far back as 1951 [2]. MS refers to an extremely severe recurrent disorder, leading to frequent hospitalizations and unsuccessful repeated treatments. It is described in pediatrics, dermatology, gastroenterology, etc. In ophthalmology, MS may lead to irreversible blindness.

Other forms of self-induced disorders concerning ocular adnexa and the eyeball should not be confused with MS [3]. In these cases, defined as 'factitious disorders', the patients' aim is not extreme self-induced mutilation leading to blindness, but more likely a desire to attract attention to themselves for other reasons.

The anterior segment involvement may be produced by mechanical (i.e. scratching, beating, rubbing) or chemical assaults (i.e. iatrogenic mechanism) $[4,5]$. 
In ophthalmic literature, various cases of unilateral or bilateral factitious disease have been described. The lesions mainly involve the more accessible anatomical eye sites: the inferior and/or temporal conjunctiva $[3,6-10]$ as well as the superior conjunctiva $[8,9]$. The cornea may be involved in some cases $[3,8,10]$ or spared $[6,7,9]$, as in our case.

The photographic documentation of this case illustrates the lesions caused in the lower part of the ocular bulb. Our case differs from the other cases described in the literature both because the entire palpebral and tarsal conjunctiva is affected, and because here the symptoms are accompanied by adolescent anorexia. Self-induced trauma is not considered a common cause of pseudo-membranous conjunctivitis [8]. Conjunctival biopsy is crucial to confirm the diagnosis; the ophthalmologist who cross-examines the patient plays a delicate role, since he is the 'discoverer of the truth'. Afterwards, a psychiatrist has to confirm the clinician's suspicion and deal with the patient.

In the case described in this report, the family completely denied any kind of familial problem.

In conclusion, in the case of chronic pseudo-membranous conjunctivitis, after having eliminated all other possible causes, the ophthalmologist should keep in mind the possibility of a self-induced lesion.
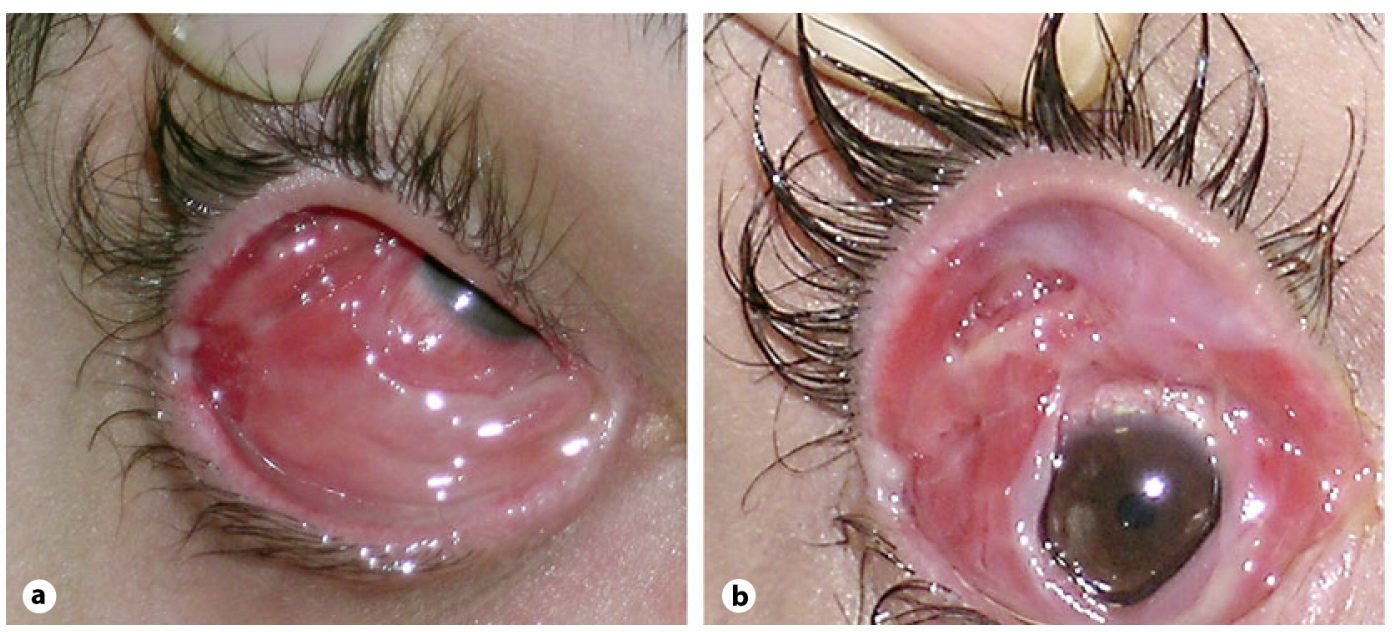

Fig. 1 a, b Right eye: inferior and superior pseudo-membranous conjunctivitis sparing the cornea. 


\begin{tabular}{l|l|l|l} 
Case Reports in & $\begin{array}{l}\text { Case Rep Ophthalmol 2011;2:69-72 } \\
\text { DOI: } 10.1159 / 000324908\end{array}$ & $\begin{array}{l}\text { Published online: } \\
\text { February 18, 2011 }\end{array}$ & $\begin{array}{l}\text { ○ 2011 S. Karger AG, Basel } \\
\text { ISSN 1663-2699 } \\
\text { www.karger.com/cop }\end{array}$ \\
\hline
\end{tabular}
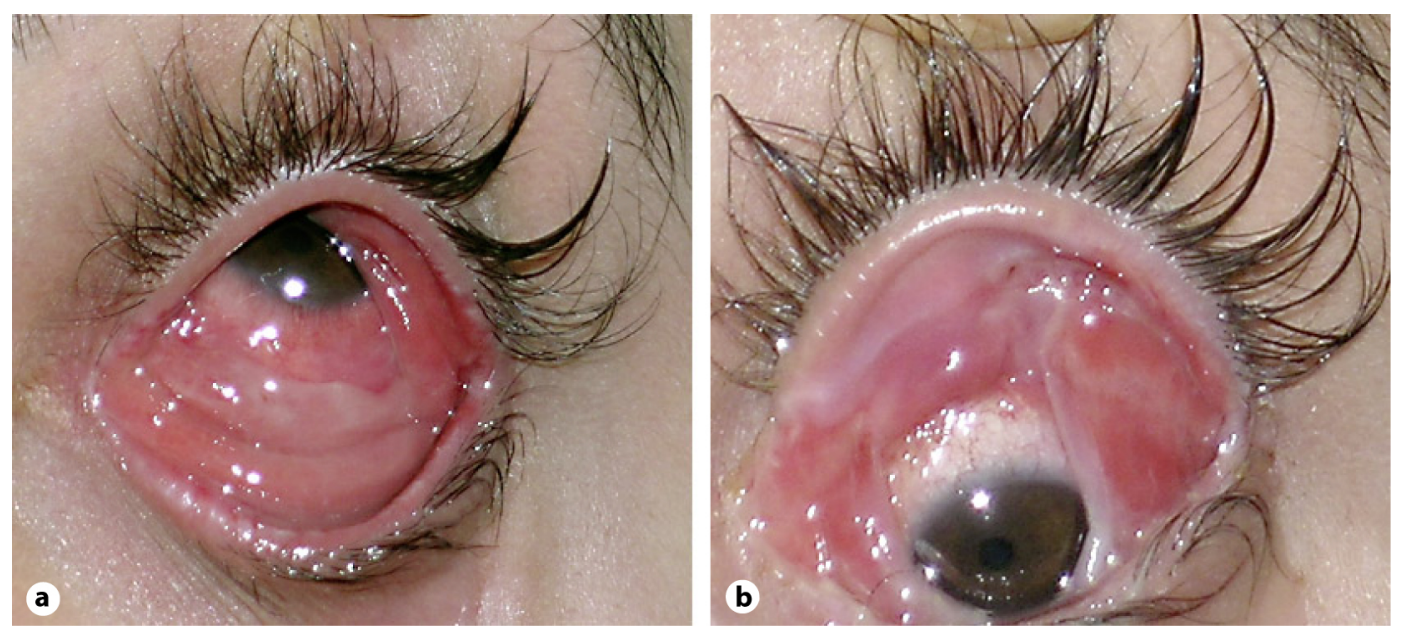

Fig. 2 a, b Left eye: inferior and superior pseudo-membranous conjunctivitis sparing the cornea.

\section{References}

1 Parsons MR: Factitious keratoconjunctivitis; in Krachmer JH, Mannis MJ, Holland EJ (eds): Cornea. Elsevier Mosby, 2005, vol 1, pp 1197-1205.

$>2$ Asher R: Munchausen's syndrome. Lancet 1951;1:339-441.

-3 Imrie FR, Church WH: Factitious keratoconjunctivitis (not another case of ocular Munchausen's syndrome). Eye 2003;17:256-258.

4 Patton N: Self-inflicted eye injuries: a review. Eye 2004;18:867-872.

5 Dart J: Corneal toxicity: the epithelium and stroma in iatrogenic and factitious disease. Eye 2003;17:886-892.

6 Pokroy R, Marcovich A: Self-induced (factitious) conjunctivitis. Ophthalmology 2003;110:790-795.

7 Braude L, Sugar J: Chronic unilateral inferior membranous conjunctivitis (factitious conjunctivitis). Arch Ophthalmol 1994;112:1488-1489.

$>8$ Kovarik GG, Hodge WG: Self-induced cicatricial conjunctivitis with symblephara. Cornea 1997;16:495-497.

-9 Chapman FM, Dickinson AJ: Self-induced cicatricial conjunctivitis. Eye 1999;13:674-676.

10 Kapoor HK, Jaison SG, Chopra R, Kakkar N: Factitious keratoconjunctivitis. Indian J Ophthalmol 2006;54:282-283. 